\title{
Feasibility and Acceptability of Anal Self-Sampling for Human Papillomavirus Screening in HIV-Infected Patients
}

\author{
Catherine Tamalet $^{\mathrm{a}}$ Isabelle Ravaux ${ }^{\mathrm{b}}$ Catherine Dhiver ${ }^{\mathrm{b}}$ Amelie Menard $^{\mathrm{b}}$ \\ Philippe Colson $^{\mathrm{a}}$ Andreas Stein $^{\mathrm{b}}$ \\ ${ }^{a}$ Fondation Institut Hospitalo-Universitaire (IHU) Méditerranée Infection, Pôle des Maladies Infectieuses et \\ Tropicales Clinique et Biologique, Fédération de Bactériologie-Hygiène-Virologie, Centre Hospitalo-Universitaire \\ Timone, and b Fondation Institut Hospitalo-Universitaire (IHU) Méditerranée Infection, Pôle des Maladies \\ Infectieuses et Tropicales Clinique et Biologique, Service de Maladies Infectieuses, Centre Hospitalo-Universitaire \\ Conception, Assistance Publique - Hôpitaux de Marseille, Marseille, France
}

\section{Keywords}

Human papillomavirus · Anal cancer screening ·

Human papillomavirus genotyping $\cdot$ High-risk human

papillomavirus types $\cdot$ Self-sampling

\begin{abstract}
Objectives: Anal cancer incidence is increasing among HIVpositive patients. No consensus currently exists for the screening of anal dysplasia. This study aimed at evaluating the feasibility and acceptability of anal self-sampling and assessing the prevalence of human papillomavirus (HPV) types among HIV-positive patients from Marseille University Hospitals. Methods: Between October 2013 and March 2014, during their regular visits for the monitoring of their HIV infection in an HIV outpatient clinical unit of Marseille University Hospitals, patients were asked to self-sample anal swabs for HPV detection. A specimen self-collection kit was provided. HPV detection and genotyping were performed using in-house protocols. The quality of self-sampling was assessed by concurrent cellular quantification in collected samples. Results: The acceptability rate of anal self-sampling was $91 \%$, and $91 \%$ of the self-sampled specimens were ap-
\end{abstract}

propriate for HPV screening. In addition, $76 \%$ of the samples were positive for HPV, including 54\% of HPV types with oncogenic potential. Conclusions: This study indicates that HPV detection and typing through anal self-sampling is a valuable strategy to screen patients at high risk for anal cancer development. This could allow earlier management of anal lesions and related cancer in patients at high risk for HPV.

c) 2016 S. Karger AG, Basel

\section{Introduction}

Human papillomaviruses (HPV) with oncogenic potential, mainly HPV type 16 (HPV16), are the major cause of cervical, anal, and vaginal cancers $[1,2]$. The incidence of anal cancer is 30-100 times higher in HIV-positive men having sex with men (MSM), in whom it ranges from 35 to 92 per 100,000 person-years in the pre- and posthighly active antiretroviral therapy (HAART) era, respectively, than in the general population, among which it ranges from 0.8 to 1.0 per 100,000 in the pre-and postHAART era, respectively $[3,4]$. In France, despite the in-

\section{KARGER}

E-Mail karger@karger.com

www.karger.com/int
(C) 2016 S. Karger AG, Base

$0300-5526 / 16 / 0592-0118 \$ 39.50 / 0$
Dr. Catherine Tamalet

IHU Méditerranée Infection, Fédération de Bactériologie-Virologie-Hygiène Centre Hospitalo-Universitaire Timone, Assistance Publique des Hôpitaux de Marseille 264 rue Saint-Pierre, FR-13385 Marseille Cedex 05 (France)

E-Mail ctamalet@ap-hm.fr 
troduction of HAART, a significant increase of the incidence of anal cancer has been observed during the 2 last decades in HIV-positive people, mostly in MSM, who have the highest risk for this neoplasia $[5,6]$.

Current French guidelines for anal cancer screening in HIV-positive patients recommend an annual digital rectal examination, combined with a standard anoscopy in at-risk patients, who include patients with anoreceptive sexual intercourse or a history of anal and/or cervical condylomas as well as women with cervical dysplasia [7]. Given the tremendous prevalence of anal HPV DNA in HIV-positive patients (85-97\%) [8, 9], routine anal HPV testing is not currently part of any recommended screening program. However, only scarce data are available on the natural history of anal HPV infection and cancer [6]. Moreover, in high-grade anal intraepithelial neoplasia, high-risk HPV and particularly HPV16 were reported to be the strongest risk factors of progression to anal cancer [10]. Hence, high-risk HPV and especially HPV16 detection on anal swabs could be used to identify patients at high risk of anal cancer, which could trigger regular monitoring and procedures such as high-resolution anoscopy and biopsy $[11,12]$. Nevertheless, anal examinations performed by clinicians can raise intimacy problems, and they are not accepted by many patients, for instance by $28 \%$ of the 142 HIV-positive MSM who were offered proctologic examination in a recent study [13]. Thus, many HIV-infected patients are not screened for anal cancer although they are at-risk individuals $[2,5]$. In the setting of cervical cancer screening, self-sampling techniques for the purpose of cervical cytology and HPV testing have been proposed as an appropriate and effective alternative to sampling performed by clinicians [14]. To date, only 3 studies have assessed anal self-sampling in HIV-positive and -negative MSM and in HIV-negative women [15-17], and 1 study has assessed feasibility, acceptability, and accuracy of anal self-sampling [18]. Here, we aimed at evaluating the acceptability and accuracy of anal self-sampling in HIV-infected patients followed-up at Marseille University Hospitals.

\section{Patients and Methods}

This study was carried out between October 2013 and March 2014. Patients who were enrolled in the study were HIV-positive individuals followed-up for their HIV infection in an HIV outpatient clinical unit of Marseille University Hospitals. During the patients' regular visits for the monitoring of their HIV infection, they were proposed to self-sample anal swabs for HPV detection. A specimen self-collection kit included a sterile Dacron swab (Mast-Diagnostic, Bootle, UK), a tube filled with Basal Medium
Table 1. Patients' characteristics and HPV results

\begin{tabular}{ll}
\hline Parameters & Results \\
\hline Sex $(\mathrm{M} / \mathrm{F})$ & $89 / 17$ \\
Median age, years & 46 \\
CD4 cell count, cells $/ \mathrm{mm}^{3}$ & $642 \pm 286$ \\
HIV RNA load, $\log _{10}$ copies/mL & $4.5 \pm 5.1$ \\
Interpretable samples & $94 / 106(89)$ \\
HPV-negative samples (positive/tested) & $23 / 94(24)$ \\
HPV-positive samples (positive/tested) & $71 / 94(76)$ \\
HPV16 & $25(35)$ \\
HPV18 & $15(21)$ \\
HPV16 + 18 & $10(14)$ \\
$\geq 2$ HPV types other than $16+18$ & $21(30)$ \\
HR HPV types & $38(54)$ \\
LR HPV types & $38(54)$ \\
\hline
\end{tabular}

Values are given as $n(\%)$ or means $\pm \mathrm{SD}$, unless otherwise indicated. HR, high-risk; LR, low-risk. ${ }^{1}$ Accurate cellular assessment for HPV detection.

Eagle medium and a transport bag. The transport bag was sent back to the laboratory by courier within $3 \mathrm{~h}$ following the sampling. The study protocol was approved by our institutional ethical board and each participant provided written informed consent. Demographic characteristics and the most recent CD4 cell count and HIV RNA load were obtained from the clinical records. HPV DNA detection, HPV16/18 DNA quantification, HPV genotyping and cellular DNA quantification were performed as previously described [19]. HPV genotyping was performed by PCR (using MY09/MY11 primers), Sanger population sequencing, and then phylogenetic analysis; in case of mixed sequence profiles, PCR products were cloned using PGEM Easy Plasmid (Promega) prior sequencing, as previously described [20]. Quantification of HPV16/18-positive samples was performed using quantitative duplex real-time PCR methods using a plasmid containing 3 target sequences of interest (E6 gene from HPV16, E7 gene from HPV 18, and exon 12 from the human albumin gene). This method allowed HPV16, HPV18, and albumin gene copy numbers to be quantified in the same assay. Therefore, HPV viral load could be expressed as HPV copies per million cells. Albumin gene quantification also enabled checking the quality of DNA, the quantity of cervical cells, and the absence of PCR inhibitors. HPV types were classified as high risk, probably high risk, and low risk according to the 2011 International Agency for Research on Cancer classification [21].

\section{Results}

Among the 116 patients invited to participate in the study and perform anal self-sampling, 106 (91\%) accepted. The mean $( \pm S D)$ age of these 106 patients was $45 \pm 10$ years (range: 22-66; median: 46), 84\% (89/106) were 
Fig. 1. Prevalence of HPV types in anal self-

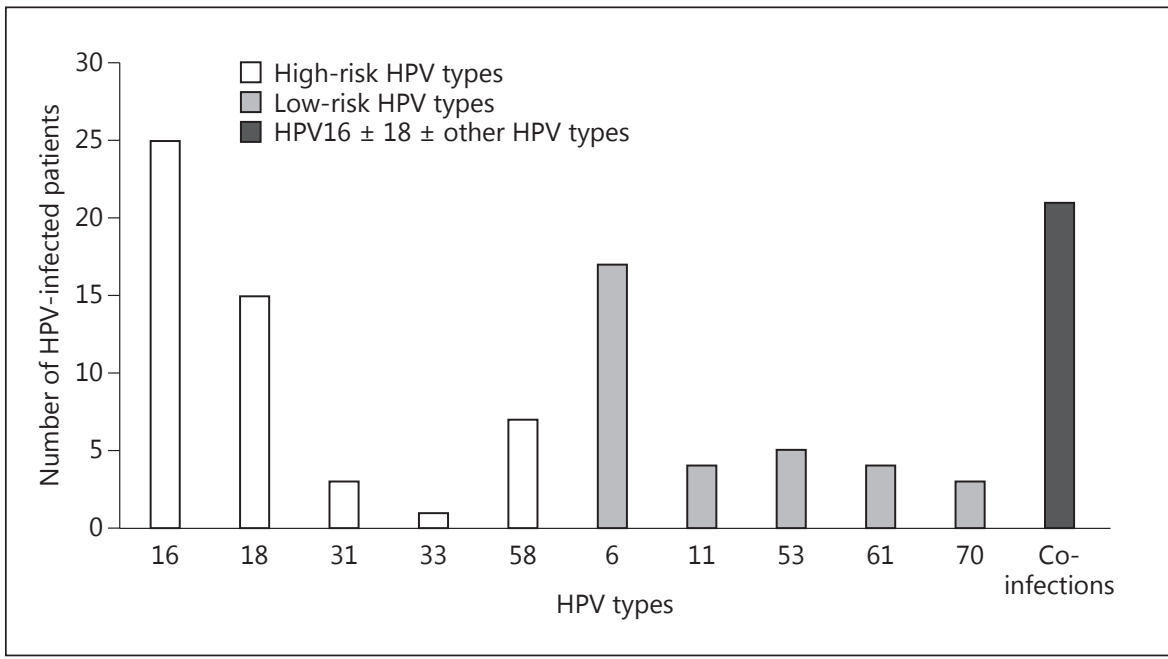
collected swabs.

male, $60 \%$ (63/106) were MSM, 20\% (21/106) were heterosexuals, $10 \%(11 / 106)$ were injecting drug users, $9 \%$ $(10 / 106)$ had no risk factor documented, and 1\% (1/106) had previously received a blood transfusion (Table 1). The mean CD4 cell count was $642 \pm 286 / \mathrm{mm}^{3}$ (range: $42-1,578$ ). The mean HIV RNA load was $4.5 \pm 5.1 \log _{10}$ copies/mL (range: 1.6-5.9). Four males and 6 females did not accept to perform anal self-sampling, including 4 MSM and 6 heterosexuals. Reasons for nonacceptance were discomfort with any anal screening, anxiety about finding an abnormality, preference for clinician collection of anal samples, and fear of hurting oneself.

Among the 106 self-collected specimens, 94 (91\%) were adequate for virological evaluation (Table 1), as assessed by albumin DNA detection with a mean CT value of 26, which is lower than the threshold CT value of 31 previously established for defining sample pauci-cellularity ( $10^{2}$ copies of albumin gene or 50 cells) [20]. Of note, the 12 pauci-cellular specimens had CT values ranging between 33 and 39. Among anal swabs with adequate cellularity self-sampled by these 94 patients, 71 (75.5\%) were HPV DNA-positive, including 38 (53.5\%) that were positive for high-risk HPV types, 38 (53.5\%) that were positive for low-risk HPV types, and $21(29.5 \%)$ that were positive for multiple HPV types (Table 1). As shown in Figure 1, the HPV types most frequently detected among the 71 HPV DNA-positive patients were HPV16 (35\%); HPV6 (24\%); HPV 18 (21\%); HPV58 (10\%); HPV53 (7\%); HPV11 and 71 (6\% each); HPV31 and 70 (4\% each); HPV62, 66, 81, and 85 (3\% each); and HPV17, 22, 33, 72, 73 , and 84 (1\% each). Twenty-one coinfections including 15 coinfections with HPV16 \pm 18 with/without other
HPV type(s) were observed. The mean \pm SD HPV16 DNA load was $7.4 \pm 8.1 \log _{10}$ copies per million cells and the mean HPV18 DNA load was $7.6 \pm 8.3 \log _{10}$ copies per million cells.

\section{Discussion}

This study is, to our knowledge, the first to assess anal self-sampling for HPV detection in a cohort of HIV-positive patients regardless of their gender and sexual behavior. We evidence here a strong acceptability (91\%) and accuracy (91\%) of anal self-sampling for HPV testing. This rate of acceptability is similar to those (90\%) reported in 2 previous studies that evaluated anal self-sampling in HIV-positive or -negative MSM $[15,16]$. In contrast, another study conducted in HIV-negative women reported a lower acceptability rate (43\%), and this was attributed to patients' concern regarding their ability to accurately collect the samples [17]. The high rate of accuracy for HPV detection via a self-sampled swab is congruent with the results from previous anal self-sampling studies that reported rates ranging between 83 and $91 \%$, either for the sake of cytological or HPV prevalence assessments $[15,22,23]$. In the present study, albumin DNA quantification in the same assay as HPV DNA detection and quantification enables to check objectively the quality of the sampling and the accuracy of the PCR testing by assessing the quantity of anal cells and the absence of PCR inhibitors, respectively. Accordingly, pauci-cellular samples could be excluded, and potential false-negative results avoided. The fact that only $10 \%$ of the anal swabs 
were not collected appropriately indicates that the anal self-sampling strategy is efficient. In addition, in the present study, the rates of detection of the main HPV types and the median HPV16 and 18 DNA loads were similar to those observed in a previous study in HIV-infected male and female patients who had anal samples collected by the clinician in another center of Marseille University Hospitals [19]. This further validates anal self-sampling for HPV detection and quantification.

With such high rates of anal self-sampling acceptability and accuracy as those observed here, this screening strategy allowed detecting high-risk HPV in one-third of the patients included, and any HPV in $61 \%$ of them. When focusing on the 94 patients who accepted anal selfsampling and collected samples with appropriate cellularity, representing $81 \%$ of those included in the study, high-risk HPV were detected in approximately half of these individuals, and any HPV were detected in approximately three quarters of them. This overall HPV prevalence $(76 \%)$ as well as the HPV16 (35\%) and $18(21 \%)$ prevalence rates found in our study are intermediate between those obtained by Lampinen et al. [23] among 222 HIV-negative MSM $(67,22$, and $8 \%$, respectively) and those found by Ortiz et al. [24] among $100 \mathrm{HIV}$-negative women whose age ranged from 18 to 34 years $(52,6$, and $2 \%$, respectively). No official guidelines currently recommend the use of HPV testing alone. However, the high prevalence of high-risk HPV in at-risk populations targeted for screening and the high prevalence of anal HPV16 in HIV-positive individuals are clinical concerns, as it was recently highlighted for HIV-infected women [6]. In addition, high-risk HPV and particularly HPV16 were reported to be the strongest risk factors of progression to anal cancer in high-grade anal intraepithelial neoplasia [10,25-27]. The present study did not aim at studying the value of HPV detection in anal cancer screening, but assessed, at a more preliminary stage, the acceptabil- ity and accuracy of anal self-sampling. An anal cytology was only available for 10 high-risk HPV-positive patients during follow-up, and 2 of them experienced low-grade dysplasia at 6 and 18 months of follow-up. The natural history of anal cancer is not fully understood. Among the few longitudinal studies conducted on the natural history of anal intraepithelial neoplasia (AIN) in a cohort of HIVseropositive patients, the rate of incident AIN2/3 over a 3 - to 4 -year period reached $34 \%$ in the study by Palefsky et al. [26] and $37 \%$ in the study by de Pokomandy et al. [28]. The exact rate of progression of high-grade AIN toward invasive carcinoma is unclear, and was estimated to be $1.3-3.2 \%$ at 5 years $[2,29]$, which justifies a long period of follow-up. In a recent study, a 2 -fold rise of the risk of progression in patients with HPV16 or 18 infections compared with patients with other HPV types was found [27]. Overall, these studies underscore the relevance of screening for AIN2/3 in HIV-positive patients and also underscore the importance of HPV typing as predictive factors for AIN2/3.

In summary, the high rates of anal self-sampling acceptability and accuracy combined with the high rate of high-risk HPV positivity in HIV-infected patients in our study indicate that such an anal self-sampling strategy is valuable for high-risk HPV detection in high-risk populations targeted for anal cancer screening. This warrants additional, larger, and more systematic studies in such patients to gain a better knowledge of the natural history of anal HPV infection and help clarify the value of HPV screening, especially HPV16, to predict the risk of anal cancer. Such an approach should be particularly fruitful in high-risk patients, and for those refusing proctologic examination by clinicians and anoscopy. In this respect, anal self-sampling might be useful in the first step of a screening algorithm. Furthermore, anal self-sampling could allow screening and treating other sexually transmitted diseases.

\section{References}

1 Arbyn M, Castellagué X, de Sanjosé S, Bruni L, Saraiya M, Bray F, Ferlay J: Worldwide burden of cervical cancer in 2008. Ann Oncol 2011;22:2675-2686.

2 Machalek DA, Poynten M, Jin F, Fairley CK, Farnsworth A, Garland SM, Hillman RJ, Petoumenos K, Roberts J, Tabrizi SN, Templeton DJ, Grulich AE: Anal human papillomavirus infection and associated neoplastic lesions in men who have sex with men: a systematic review and meta-analysis. Lancet Oncol 2012;13:487-500
3 Bower M, Powles T, Newsom-Davis T, Thirlwell C, Stebbing J, Mandalia S, Nelson M, Gazzard B: HIV-associated anal cancer: has highly active antiretroviral therapy reduced the incidence or improved the outcome? J Acquir Immune Defic Syndr 2004;37:15631565.

4 Chiao EY, Known SE, Stier EA, Schrag D: A population-based analysis of temporal trends in the incidence of squamous anal canal cancer in relation to the HIV epidemic. J Acquir Immune Defic Syndr 2005;40:451-455. 
5 Piketty C, Selinger-Leneman H, Grabar S, Duvivier C, Bonmarchand M, Abramowitz L, Costagliola D, Mary-Krause MP: Marked increase in the incidence of invasive anal cancer among HIV-infected patients despite treatment with combination antiretroviral therapy. AIDS 2008;22:1203-1211.

6 Heard I, Poizot-Martin I, Potard V, Etienney I, Crenn-Hebert C, Moore C, Touraine P, Cubie $\mathrm{H}$, Costagliola D: Prevalence of and risk factors for anal oncogenic human papillomavirus infection among HIV-infected women in France in the combination antiretroviral therapy era. J Infect Dis 2016;213:455-461.

7 Prise en charge des personnes vivant avec le VIH. Recommandations du groupe d'experts Rapport 2013. La Documentation Française. http://social-sante.gouv.fr/IMG/pdf/Rapport_Morlat_2013_Mise_en_ligne.pdf.

8 Vajdic CM, Van Leeuwen MT, Jin F, Prestage G, Medley G, Hillman RJ, Stevens MP, Botes LP, Zablotska I, Tabrizi N, Grulich AE: Anal human papillomavirus genotype diversity and co-infection in a community-based sample of homosexual men. Sex Transm Infect 2009;85:330-335.

9 Damay A, Fabre J, Costes V, Didelot JM, Didelot MN, Boulle N, Segondy M: Human papillomavirus (HPV) prevalence and type distribution, and HPV-associated cytological abnormalities in anal specimens from men infected with HIV who have sex with men. J Med Virol 2010;82:592-596.

10 Burd EM: Human papillomavirus laboratory testing: the changing paradigm. Clin Microbiol Rev 2016;29:291-318.

11 Ortoski RA, Kell CS: Anal cancer and screening guidelines for human papillomavirus in men. J Am Osteopath Assoc 2011;111(suppl 2):S35-S43.

12 Leeds IL, Fang SH: Anal cancer and intraepithelial neoplasia screening: a review. World J Gastrointest Surg 2016;8:41-51.

13 Read TRH, Vodstrcil L, Grulich AE, Farmer C, Bradshaw CS, Chen MY, Tabrizi S, Hocking JS, Anderson J, Fairley CK: Acceptability of digital anal cancer screening examinations in HIV-positive homosexual men. HIV Med 2013;14:491-496.
14 Wright TC, Denny L, Kuhn L, Pollack A, Lorincz A: HPV DNA testing of self-collected vaginal samples compared with cytologic screening to detect cervical cancer. JAMA 2000;283:81-86.

15 Cranston RD, Darragh TM, Holly EA, Jay N, Berry JM, Da Costa M, Efird JT, Palefsky JM: Self-collected versus clinician-collected anal cytology specimens to diagnose anal intraepithelial neoplasia in HIV-positive men. J Acquir Immune Defic Syndr 2004;36:915-920.

16 Rosenberg JG, Dodge B, Van der Pol B, Reece $M$, Herbenick D, Fortenberry JD: Reactions to self-sampling for ano-rectal sexually transmitted infections among men who have sex with men: a qualitative study. Arch Sex Behav 2011;40:281-288.

17 Ortiz AP, Alejandro N, Pérez CM, Otero Y, Soto-Salgado M, Palefsky JM, Tortolero-Luna G, Romaguera J: Acceptability of cervical and anal HPV self-sampling in a sample of Hispanic women in Puerto Rico. PR Health Sci J 2012;31:205-212.

18 Chin-Hong PV, Berry JM, Cheng SC, Catania JA, Da Costa M, Darragh TM, Fishman F, Jay N, Pollack LM, Palefsky JM: Comparison of patient- and clinician-collected anal cytology samples to screen for human papillomavirusassociated anal intraepithelial neoplasia in men who have sex with men. Ann Intern Med 2008;149:300-306.

19 Tamalet C, Obry-Roguet V, Ressiot E, Bregigeon S, Del Grande J, Poizot-Martin I: Distribution of human papillomavirus genotypes, assessment of HPV16 and 18 viral load and anal related lesions in HIV positive patients: a cross-sectional analysis. J Med Virol 2014;86: 419-425.

20 Tamalet C, Richet H, Carcopino X, Henry M, Leretraite L, Heid P, Leandri FX, Sancho-Garnier H, Piana L: Testing for human papillomavirus and measurement of viral load of HPV16 and 18 in self-collected vaginal swabs of women who do not undergo cervical cytological screening in Southern France. J Med Virol 2010;882:1431-1497.

21 Bouvard V, Baan R, Straif K, Grosse Y, Secre$\tan$ B, El Ghissassi E, Benbrahimi-Tallaa L, Guha N, Freeman C, Galichet L, Cogliano V: A review of human carcinogens. Part B: biological agents. Lancet Oncol 2009;10:321322.
22 Lampinen TM, Latulippe L, Van Niekerk D, Schilder AJ, Miller ML, Anema A, Hogg RS: Illustrated instructions for self-collection of anorectal swab specimens and their adequacy for cytological examination. Sex Transm Dis 2006a;33:6:386-388.

23 Lampinen TM, Chan K, Anema A, Kornegay J, Hogg RS, Coutlée F: Self-screening for rectal sexually transmitted infections: human papillomavirus. Clin Infect Dis 2006;42:308-309.

24 Ortiz AP, Romaguera J, Pérez C, Otero Y, Soto-Salgado M, Mendez K, Valle Y, Da Costa M, Suarez E, Palefsky J, Tortolero-Luna G: Human papillomavirus infection in women in Puerto Rico: agreement between physiciancollected and self-collected anogenital specimens. J Low Genit Tract Dis 2013;17:210224.

25 Park IU, Palefsky JM: Evaluation and management of anal intra-epithelial neoplasia in HIV-negative and HIV-positive men who have sex with men. Curr Infect Dis Rep 2010; 12:126-123.

26 Palefsky JM, Holly EA, Hogeboom JC, Ralston ML, DaCosta MM, Botts R, Berry JM, Jay N, Darragh TM: Virologic, immunologic, and clinical parameters in the incidence and progression of anal squamous intraepithelial lesions in HIV-positive and HIV-negative homosexual men. J Acquir Immune Defic Syndr Hum Retrovirol 1998;17:314-319.

27 Burgos J, Curran A, Tallada N, Guelar A, Navarro J, Landolfi S, Villar J, Crespo M, Ribera E, Falco V: Risk of progression to high-grade anal intraepithelial neoplasia in HIV-infected MSM. AIDS 2015;29:695-702.

28 de Pokomandy A, Rouleau D, Ghattas G, Trottier H, Vézina S, Coté, $\mathrm{P}$, Macleod J, Allaire G, Hadjeres R, Franco EL, Coutlée F: HAART and progression to high-grade anal intraepithelial neoplasia in men who have sex with men and are infected with HIV. Clin Infect Dis 2011;52:1174-1181.

29 Cachay ER, Mathews WC: Human papillomavirus, anal cancer, and screening considerations among HIV-infected individuals. AIDS Rev 2013;15:122-133. 